\title{
Bilateral simultaneous multifocal primary tuberculous pyomyositis in a renal transplant recipient - a rare presentation
}

\author{
L N Senevirathna, B Lindsey, D Nicol \\ Department of Nephrology, Urology \& Renal Transplant, Royal Free Hospital, London, \\ UK
}

Key words: Pyomyositis; acid fast bacilli; renal transplantation

\section{Introduction}

Tuberculosis (TB) is a serious infection with high mortality of $22-31 \%$ in solid organ transplantation which is higher than in the general population [1]. Primary infection is rare and in the majority of cases $\mathrm{TB}$ is due to reactivation of latent infection. The diagnosis of TB in renal transplant recipients is a diagnostic dilemma due to the atypical and extra pulmonary presentations. Primary muscular TB without the involvement of the bones is rarer and limited to case reports [2]. This is mainly due to the fact that striated muscle is most resistant to the bacteria because of its poor oxygen content, high lactic acid and a paucity of reticuloendothelial tissue [3].

\section{Case report}

A 29-year-old man who was well following a live donor renal transplant performed three years back presented with a complaint of thigh pain and pain on walking for two weeks. Examination was unremarkable with normal neurovascular status of the limbs. Routine investigations including muscle enzymes were normal. Worsening myalgia warranted a muscle biopsy which showed non specific inflammatory changes. A few weeks later the pain worsened with induration of the skin and the subcutaneous tissues of the thigh. CT showed no bony destruction but revealed multiloculated heterogeneous collections in the posterior compartments of both thighs (Fig 1). MRI showed

Correspondence :

Seneviratne LN

Email : neerosene@yahoo.com extensive posterior compartmental, inter and intra muscular abscesses occupying the region from the gluteus maximus to the hamstrings with high signal on T2 -weighted images (Fig 2). Chest radiograph was normal. Due to the extensive nature of the abscess open surgical drainage was done via bilateral small vertical skin incisions with extensive subcutaneous fasciotomy (Figure 3). Bacterial and fungal cultures and microscopy for AFB were negative. However PCR for TB was positive. Extended course of antituberculous drugs was given resulting a slow, steady recovery.

\section{Discussion}

This is the first reported case of primary TB involving both lower limbs involving the same muscular compartment simultaneously. TB pyomyositis has been documented in both immunocompetent individuals via syringe transmitted infections and in immunocompromised patients such as in HIIV, systemic lupus erythematosus, leukaemia and transplanted patients. ${ }^{4}$ The classic inflammatory signs are absent, making an early diagnosis difficult. Low grade fever and myalgia are less common and the finding of a non tender mass is the most common clinical sign.

The pathogenesis of primary muscular TB has not been clearly established. All published reports of primary TB of gluteal muscles cite that contaminated syringes were as the source. Haematogenous spread and direct extension from lung, bone or other tissues are potential mechanisms. In this case we found no other primary focus nor was there a history of trauma or injections. The pathogenesis remained a mystery.

The muscle groups commonly involved are the psoas, 
The Sri Lanka Journal of Surgery 2012; 30(1)

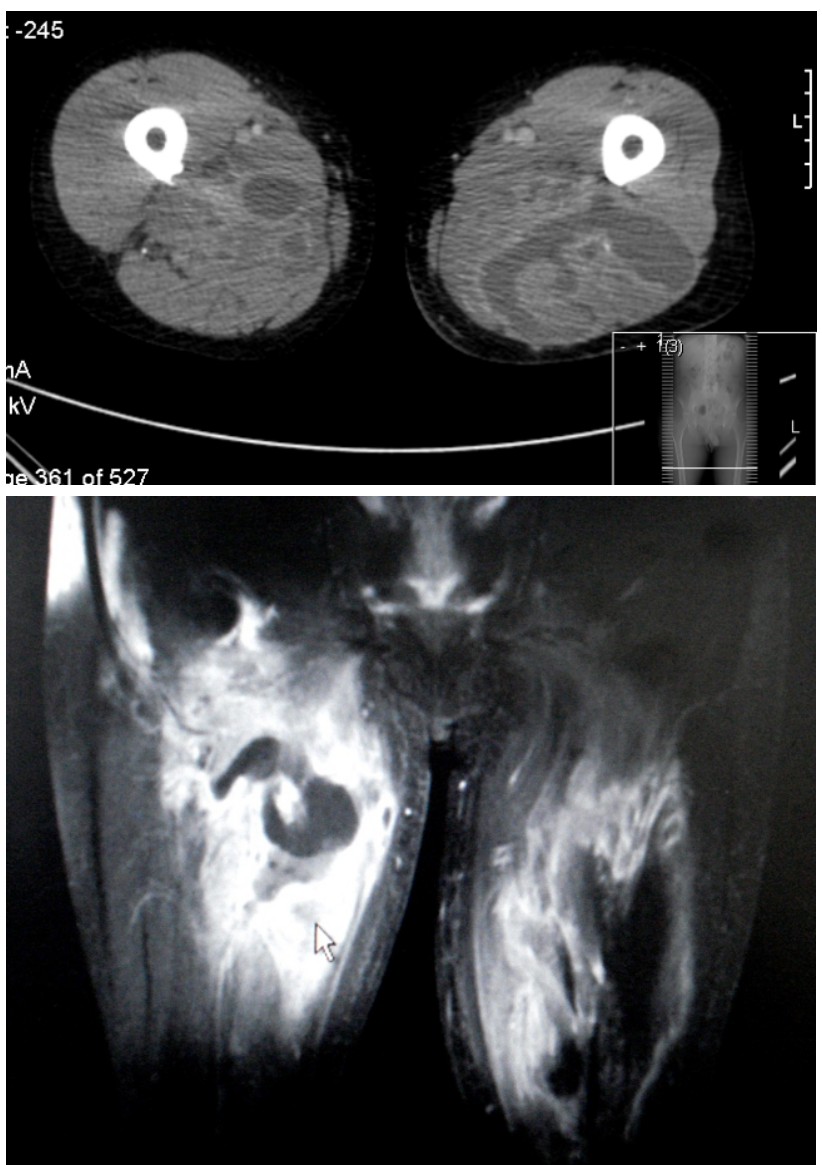

Figure la: CI scan, Figure lb: MIRI scan of both

biceps, and gluteus maximus.

Interestingly this patient had the entire posterior compartmental muscles involved including glutei and hamstrings. Also surprisingly both limbs were involved more or less to the same extent. Interestingly, although extensive myofibrillar destruction occurs in pyomyositis, it is uncommon to see muscle enzyme elevation, and normal enzyme levels do not exclude the diagnosis as highlighted in this case.

CT, MRI and scintigraphic scanning with gallium-67 can be helpful in the diagnosis of occult abscesses. MR imaging is more accurate in identifying and evaluating the loco-regional extension of pyomyositis. Gadolinium enhancement during MRI, if performed early will differentiate the pre- abscess invasive stage from the suppurative stage.

CT guided percutaneous drainage of the the abscess is as effective for both diagnosis and treatment in early stages. In this case CT guided drainage was not
A case of undetected congenital diaphragmatic hernia in an adult

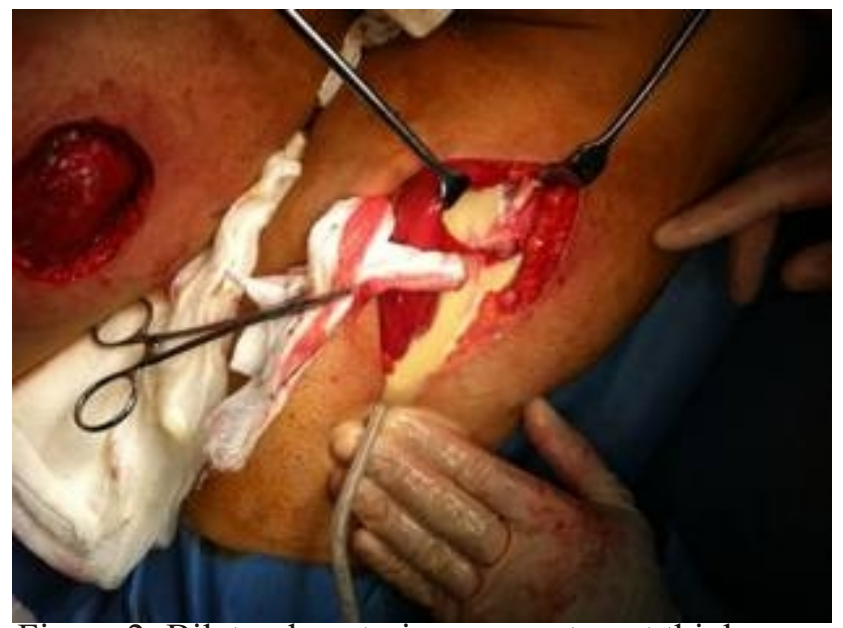

Figure 2: Bilateral posterior compartment thigh

considered due to the multiloculated and extensive nature of the lesion.

Standard antituberculous treatment yields a good response in most cases. This case highlights the importance of having a high index of suspicion of extrapulmonary TB and having a low threshold for imaging since it is the corner stone to making the an early diagnosis in order to prevent significant morbidity.

\section{References}

1.Hsu MS, Wang JL, Ko WJ et al. Clinical features and outcome of tuberculosis in solid organ transplant recipients. Am J Med Sci 2007;334(2):106-110

2.Trikha V, Gupta V. Isolated tuberculous abscess in biceps brachii muscle of a young male. J Infect 2002;44:265-6

3.Kim JY, Park YH, Choi KH, Park SH, Lee HY. MRI of tuberculos pyomyositis. J Comput Assist Tomogr 1999;23:454-7

4. Jhonson DW, Herzig KA: Isolated tubeculos pyomyositis in renal transplant paient. Nephrol Dial Transplant 2000; 15:543

5.Kim JY, Park YH, Choi KH, Park SH, Lee HY. MRI of tuberculous pyomyositis. J Comput Assist Tomogr 1999;23:454-457 